\title{
Performance of the ATLAS Level-1 muon barrel trigger during the Run 2 data taking
}

\author{
Marco Sessa* \\ On behalf of the ATLAS Collaboration \\ Università degli Studi Roma Tre, Dipartimento di Matematica e Fisica \\ INFN, sezione Roma Tre \\ CERN \\ E-mail: marco.sessadcern.ch
}

The Level-1 Muon Barrel Trigger is one of the main elements of the online event selection of the ATLAS experiment at the Large Hadron Collider. It exploits the Resistive Plate Chambers (RPC) detectors to generate the trigger signal. The RPCs are placed in the barrel region of the ATLAS experiment: they are arranged in three concentric double layers and operate in a strong magnetic toroidal field. RPC detectors cover the pseudo-rapidity range $|\eta|<1.05$ for a total surface of more than $4000 \mathrm{~m}^{2}$ and about 3600 gas volumes. The Level-1 Muon Trigger in the barrel region allows to select muon candidates according to their transverse momentum and associates them with the correct bunch-crossing. The trigger system is able to take a decision within a latency of about $2 \mu$ s. The measurement of the RPC detector efficiencies and the trigger performance during the ATLAS Run 2 data taking are here presented.

The European Physical Society Conference on High Energy Physics

5-12 July, 2017

Venice

${ }^{*}$ Speaker. 


\section{The ATLAS RPC Muon Barrel System}

The present ATLAS [1] muon trigger in the barrel region $(|\eta|<1.05)$ is based on three concentric layers of Resistive Plate Chambers (RPCs). RPCs operate in a strong magnetic toroidal field and cover a surface area of about $4000 \mathrm{~m}^{2}$. RPC1 and RPC2 stations are located in the Medium Layer (BM) of the Muon Spectrometer (MS), while the third station, RPC3, is in the Outer Layer (BO). Each RPC detector consists of two gas gaps ( $2 \mathrm{~mm}$ width), read out by two orthogonal planes of strips, in $\eta$ and $\phi$ views, with a width of $23-35 \mathrm{~mm}$.

In the MS, the Barrel Toroid magnet, consisting of eight super-conducting coils, generates a magnetic field of about $0.5 \mathrm{~T}$ and causes muon trajectories to bend in the $\eta-z$ plane $^{1}$, needed for muon transverse momentum measurement. RPCs are assembled together with Monitored Drift Tube (MDT) chambers, which are used for precise muon tracking in the bending direction only. RPC detectors are hence used to provide the $\phi$ coordinate measurement in the barrel MS.

Since the inner layer of the MS is currently not instrumented with RPCs, it has been proposed to increase the current detector coverage using a new type of RPCs, in view of High Luminosity LHC.

\section{The muon barrel trigger logic}

The Level-1 (L1) trigger algorithm is based on the coincidence of hits from three concentric RPC stations (both in $\eta$ and $\phi$ projections). Two different $p_{\mathrm{T}}$-regimes exist:

- the low- $p_{\mathrm{T}}$ trigger requires a coincidence between the innermost two RPC stations (BM). It is used to select muons with $p_{\mathrm{T}}$ above the three thresholds of $4 \mathrm{GeV}, 6 \mathrm{GeV}$ and $10 \mathrm{GeV}$;

- the high- $p_{\mathrm{T}}$ trigger requires an additional confirmation on the third external station (BO) and selects muons with $p_{\mathrm{T}}$ above the three thresholds of $11 \mathrm{GeV}, 15 \mathrm{GeV}$ and $20 \mathrm{GeV}$ up to 2016 and $11 \mathrm{GeV}, 20 \mathrm{GeV}$ and $21 \mathrm{GeV}$ since $2017^{2}$.

During ATLAS Run 2, only the high- $p_{\mathrm{T}}$ triggers are used for single-muon signatures, while the low- $p_{\mathrm{T}}$ triggers are used in coincidence with other trigger objects to select multi-object signatures, including muon pairs.

One of the main requirements of the $\mathrm{L} 1$ trigger system is the association of the triggering muon to the correct collision bunch crossing (BC). Fig.1 (left) shows the time distribution (2.9 ns width) of the trigger hits associated to reconstructed muons, compared to the interval corresponding to the collision BC. Fig.1 (right) shows the fraction of RPC trigger hits associated correctly to the collision $\mathrm{BC}$ for each of the muon barrel trigger towers. The fraction of reconstructed muons with $p_{\mathrm{T}}>10 \mathrm{GeV}$ associated to the correct $\mathrm{BC}$ is $99.7 \%$ [2].

\footnotetext{
${ }^{1}$ ATLAS experiment uses a right-handed coordinate system. The origin of the system is in the nominal protonproton interaction point, set in the center of the ATLAS detector. The $\mathrm{z}$ axis is along the beam line, while the xy plane is the plane perpendicular with respect to the beam line. The positive $\mathrm{x}$-axis is defined as pointing from the interaction point to the centre of the LHC ring and the positive y-axis is defined as pointing upwards.

${ }^{2}$ MU21 threshold is equal to MU20 (same threshold on muon $p_{\mathrm{T}}$ at $20 \mathrm{GeV}$ ) everywhere but in the "feet" region. The new feet trigger, shown in Section 3, does not have the MU21 threshold. This distinction is done to separately monitor the trigger rate with (MU20) and without (MU21) the new feet trigger.
} 

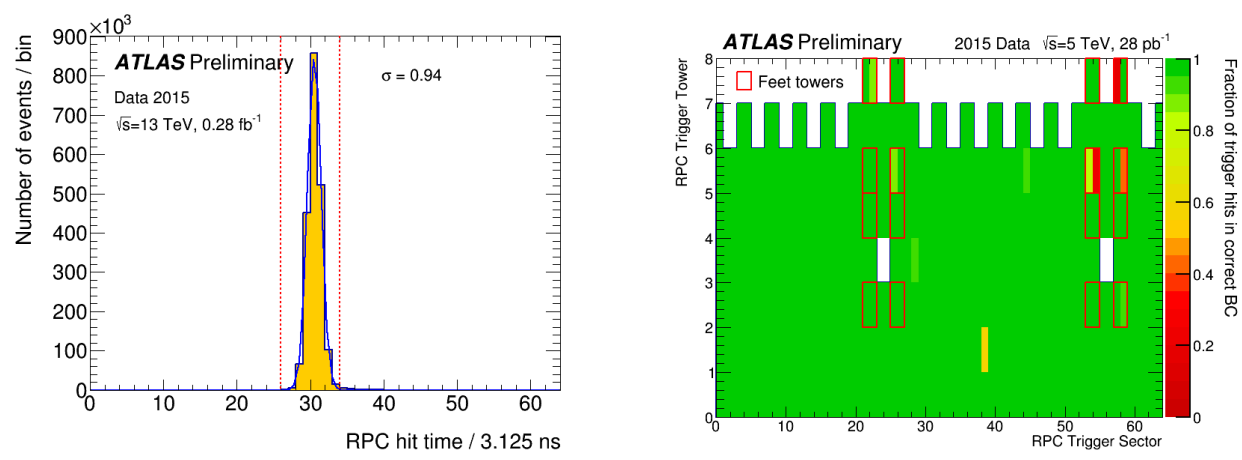

Figure 1: Time distribution of the trigger hits associated to reconstructed muons (left) and fraction of RPC trigger hits associated correctly to the collision BC for each trigger tower (right) [3].

\section{RPC "feet" sector trigger upgrade}

In order to avoid the acceptance losses of about $3 \%$ in the "feet" regions ${ }^{3}$ of the detector [2], additional RPC chambers have been installed in 2006 in sectors $12(-2.16<\phi<-1.77)$ and 14 ( $-1.37<\phi<-0.98)$ in a new $4^{\text {th }}$ layer in projective geometry with respect to the interaction point. At that time, the trigger electronics was not yet available. It was installed later during the Long Shutdown 1 (LS1) between 2013 and 2014. At the beginning of 2015, the commissioning of the new feet trigger started and it was concluded at the end of the same year.

The efficiency increase in the feet sectors, due to the deployment of the new trigger RPC chambers, has been evaluated to be about $20 \%$. The efficiency is computed on an inclusive sample, selected using all non-muon L1 ATLAS triggers, obtained from 2016 data-taking at $\sqrt{s}=13 \mathrm{TeV}$. Muons are required to pass "Medium" quality requirements [4] and have a transverse momentum of at least $15 \mathrm{GeV}$. In Fig.2 (left), the efficiency is plotted as a function of the azimuthal angle $\phi$ of the muon candidates in the barrel detector region. In Fig.2 (right), the trigger efficiency as a function of the muon pseudo-rapidity $\eta$ is shown for a specific feet sector.
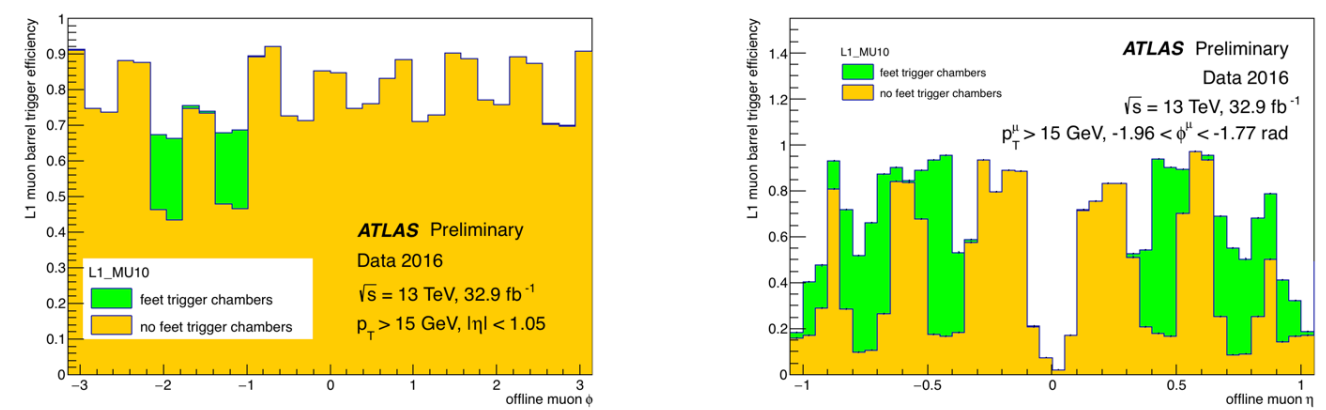

Figure 2: Trigger efficiency as a function of the azimuthal angle $\phi$ of the muon candidates in the barrel detector region (left) and trigger efficiency as a function of the muon pseudo-rapidity for a specific feet sector (right) [3].

\footnotetext{
${ }^{3}$ The feet system is the main support of the ATLAS detector. It is made of nine pairs of feet, bound by girders that altogether support the two bottom coils of the barrel toroid magnet. This region corresponds to the Sectors 12 and 14 of the Muon Spectrometer.
} 


\section{RPC “elevator" chamber upgrade}

To cover the $\sim 1 \%$ acceptance holes corresponding to two elevator shafts in the ATLAS Muon Spectrometer, new chambers consisting of two layers of RPC detectors were installed during 2014. The BME chambers, located in the Medium Layer, are the first large size RPC chambers of a new type (1 mm gas gaps); the BOE chambers consist of standard RPCs and are located in the Outer Layer. Since this new BME chamber technology will be employed for the upgrades of the Muon Spectrometer for High Luminosity LHC, the test of their performance during ATLAS Run 2 is an essential step. New data have shown that the new chambers are fully working. Trigger commissioning is still ongoing in order to optimise the efficiency.

\section{Trigger performance with early 2017 data}

The efficiency of the muon barrel trigger is measured using a tag-and-probe method with $Z \rightarrow \mu \mu$ candidates, with no background subtraction applied, in $13 \mathrm{TeV}$ data from 2017. The plots in Fig.3 show the absolute efficiency of the L1 MU20 trigger and the absolute and relative efficiencies of the OR of mu26 (isolation requirement dependent on the muon $p_{\mathrm{T}}$ is also applied here) with mu60 High Level Triggers (HLT), plotted as a function of $p_{\mathrm{T}}$ and $\phi$ of off-line muon candidates in the barrel detector region. Reconstructed muons are required to pass "Medium" quality requirements [4].

The L1 trigger inefficiency is due mainly to the detector acceptance in the barrel region $(\sim 80 \%)$. The residual inefficiency is ascribed to malfunctioning detector elements. Globally, the system shows very good purity, with more than $90 \%$ of the L1 triggers that correspond to a good muon reconstructed off-line and originating from the interaction region [2].
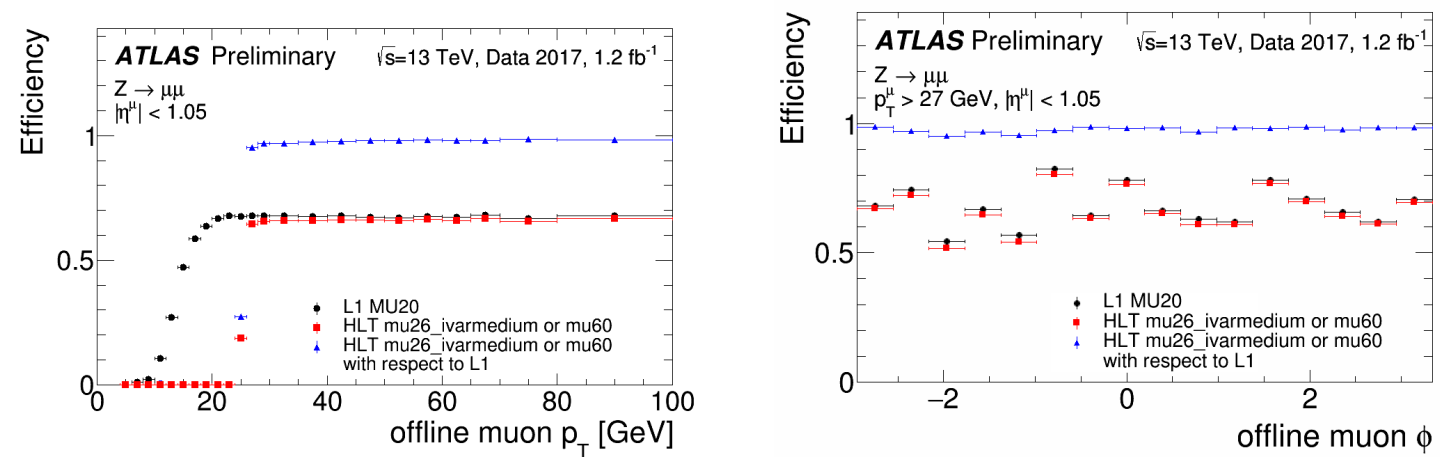

Figure 3: Absolute efficiency of the L1 MU20 trigger and absolute and relative efficiencies of the OR of mu26 with mu60 High Level Triggers, plotted as a function of $p_{\mathrm{T}}$ (left) and $\phi$ (right) of off-line muon candidates [5].

\section{References}

[1] ATLAS Collaboration, 2008 JINST 3 S08003

[2] M. Corradi, Performance of ATLAS RPC Level-1 muon trigger during the 2015 data taking, 6032 Journal of Instrumentation 11 (2016) C09003

[3] https://twiki.cern.ch/twiki/bin/view/AtlasPublic/L1MuonTriggerPublicResults

[4] ATLAS Collaboration, Muon reconstruction performance of the ATLAS detector in proton-proton collision data at $\sqrt{s}=13 \mathrm{TeV}$, Eur. Phys. J. C (2016) 76: 292.

[5] https://twiki.cern.ch/twiki/bin/view/AtlasPublic/MuonTriggerPublicResults 